\title{
Cytokines d'origine adipocytaire, obésité et développement du diabète
}

> La régulation et le maintien d'un état métabolique normal dépend d'un système d'homéostasie complexe incluant de nombreux organes et tissus. Des défauts acquis dans ce système associés à des facteurs environnementaux mènent au développement d'anomalies métaboliques comme le syndrome $X$, qui est devenu un syndrome fréquent

Corinne Lacquemant, Francis Vasseur, Frédéric Leprêtre, Philippe Froguel dans les pays industrialisés. Ce syndrome se caractérise par l'association d'anomalies incluant une résistance à l'insuline, un hyper-insulinisme, une intolérance au glucose ou un diabète de type 2 , une hypertension artérielle et des troubles du métabolisme lipidique et de la coagulation. Sa gravité résulte de la fréquence des complications cardiovasculaires associées à ces altérations. La résistance à l'insuline du tissu adipeux (en particulier viscéral) et du muscle, qui se répercute sur le métabolisme hépatique, et les altérations des flux de substrats métaboliques entre ces tissus jouent un rôle majeur dans sa physiopathologie. De nouvelles classes pharmacologiques viennent enrichir l'arsenal thérapeutique et semblent prometteuses pour traiter l'insulinorésistance. À cet égard, le tissu adipeux n'est plus simplement considéré comme un simple tissu de stockage des réserves graisseuses, mais comme un organe jouant un rôle actif dans la régulation métabolique. Le tissu adipeux sécrète, en effet, une grande variété de molécules biologiquement actives agissant pour prévenir l'accumulation délétère de lipides et la modulation de l'insulinorésistance. Cet article a pour objectif de mieux comprendre les mécanismes menant à l'insulinorésistance et au diabète de type 2 , selon les plus récentes avancées dans la connaissance du rôle et des interactions d'hormones spécifiquement issues de l'adipocyte, récemment identifiées, et appelées adipocytokines ou adipokines. <

L'étude des adipokines, protéines de la famille des cytokines produites par le tissu graisseux, est d'actualité. Longtemps considéré comme un simple tissu de stockage des réserves graisseuses dont les débordements contribueraient à aggraver un syndrome métabolique d'insulinorésistance d'origine différente, le tissu adipeux est aussi un organe endocrine qui sécrète de nombreuses protéines interagissant avec les métabolismes glycémique et lipidique. On connaît la leptine, facteur de contrôle de la prise alimentaire, le facteur de nécrose tumorale $\alpha$ (TNF $\alpha$ ), l'interleukine 6 et, plus récemment, la mystérieuse résistine, qui induisent une insulinorésistance quand ils sont produits en excès. De plus, de nombreux résultats récents ont révélé l'existence d'une hormone sécrétée, circulant en abondance, appelée adiponectine ou ACRP30 (adipocyte complement rela- 
ted protein of $30 \mathrm{kDa}$ ). La combinaison des études de génomique animale et humaine a permis d'établir l'importance de cette protéine dans la «protection» contre l'insulinorésistance et dans la transition métabolique entre l'obésité et le diabète de type 2. Les effets contradictoires de ces adipokines illustrent la double nature de notre patrimoine graisseux : indispensable à une vie normale, son excès est un des maux modernes liés au développement de ces pathologies.

\section{L'adiponectine : un modulateur du métabolisme énergétique induit par le tissu graisseux}

L'adiponectine, cytokine sécrétée par le tissu adipeux, est une protéine plasmatique environ 1000 fois plus abondante que la résistine. Elle a été identifiée et caractérisée de manière indépendante et simultanée par plusieurs groupes, chez l'homme comme chez la souris. Cela explique ses diverses appellations dans la littérature : adipoQ, par analogie structurale avec la fraction $\mathrm{Clq}$ du complément et des cytokines de type TNF, ACRP30 ou GBP28 (gelatin binding protein 28). Chez l'homme, il s'agit d'une protéine de 244 acides aminés porteuse d'un domaine globulaire et d'un domaine de type collagène. L'adiponectine est codée par le gène APMI (adipose most abundant transcript 1) qui est exclusivement exprimé dans l'adipocyte. Ce gène, composé de 3 exons dont le premier est non codant, est situé sur le chromosome 3 en 3 q27.

Dès sa découverte par Scherer en 1995, le rôle de l'adiponectine dans l'homéostasie énergétique a été suspecté [1] du fait de la modulation de sa sécrétion par l'insuline. Nakano suspectait aussi son implication dans le catabolisme des lipides [2]. II a fallu attendre les années 2000 pour que la relation étroite entre insulinorésistance et adiponectine soit métaboliquement confirmée et ses bases moléculaires en partie expliquées. Chez l'homme, l'adiponectinémie est corrélée négativement à l'indice de masse corporelle (IMC) [3]. Les situations pathologiques associant une insulinorésistance au diabète de type 2 et à l'obésité, comme c'est le cas de la lipodystrophie, présentent des taux sériques d'adiponectine effondrés. Cela suggère que l'adiponectine pourrait être non seulement un marqueur de sensibilité à l'insuline, mais aussi un lien métabolique entre l'activité du tissu adipeux et l'insulinorésistance.

Par ailleurs, il existe un lien étroit entre l'insulinorésistance et l'athérosclérose. Les taux d'adiponectine sont encore plus réduits chez les patients souffrant d'un diabète de type 2 compliqué d'athérosclérose
[4]. D'autres études suggèrent un rôle «anti-inflammatoire» direct de l'adiponectine sur la paroi vasculaire. Cette adipokine, qui s'accumule dans la paroi vasculaire lésée, exerce un effet inhibiteur sur la production de TNF $\alpha$ par les macrophages [5]. De plus, l'adiponectine module la réponse inflammatoire des cellules endothéliales par inhibition du signal engendré par le facteur nucléaire NFKB et réduction de l'adhésion et de l'expression induite par le TNF $\alpha$ des molécules endothéliales VCAM-1 (vascular cell adhesion molecule-1) et ICAM-1 (intercellular adhesion molecule-1) [6]. Dans les macrophages humains dérivés des monocytes, l'adiponectine supprime l'accumulation de lipides et l'expression du récepteur scavenger de classe $A$, ce qui suggère un rôle modulateur de l'adiponectine dans la transformation des macrophages en cellules spumeuses [7]. Récemment, un effet modulateur du remodelage vasculaire a également été suggéré par l'activité suppressive de l'adiponectine sur la prolifération et la migration des cellules musculaires lisses aortiques humaines. Un modèle de souris déficiente en adiponectine a montré une formation néointimale, en réponse à une lésion vasculaire externe, deux fois plus importante $(p=0,01)$ que celle observée chez des souris normales [8]. De plus, ce type de souris présentait une insulinorésistance modérée accompagnée d'intolérance au glucose. Par ailleurs, le traitement des souris déficientes en adiponectine par un adénovirus produisant de l'adiponectine atténuait la prolifération néointimale. L'adiponectine semble donc être une cytokine à effet «anti-insulinorésistance» et «anti-athérosclérose». D'ailleurs, il existe un dimorphisme sexuel quant à la sécrétion d'adiponectine dont les taux, diminués par les hormones androgènes, sont plus élevés chez la femme. Ce dimorphisme pourrait contribuer à expliquer la plus grande susceptibilité masculine à l'insulinorésistance liée à l'obésité androïde et au risque coronarien précoce.

Si la variation des taux sériques d'adiponectine est étroitement liée à la sensibilité à l'insuline chez I'homme, la relation causale entre ces deux événements reste à démontrer. Cependant, la diminution des taux d'adiponectine semble précéder l'apparition de l'insulinorésistance, et «prédit» mieux que la glycémie et l'insulinémie la progression vers le diabète de type 2 [9]. Une perte de poids chez des sujets obèses accompagnée d'une amélioration de l'insulinosensibilité entraînent une augmentation des taux plasmatiques d'adiponectine. De plus, les effets insulinosensibilisants des nouvelles molécules antidiabétiques comme les agonistes du récepteur nucléaire PPAR $\gamma$ (thiazolinediones) s'ac- 
compagnent d'une élévation de l'adiponectinémie. Par ailleurs, les porteurs du variant Prol2Ala du gène PPAR $\gamma$, dont on sait qu'il est protecteur vis-à-vis du diabète de type 2 , ont une adiponectinémie élevée. À l'inverse, les patients diabétiques, insulinorésistants et hypertendus, porteurs de mutations à effet dominant négatif de PPAR $\gamma$ ont une adiponectinémie effondrée. Ainsi, chez l'homme, l'hypoadiponectinémie est un facteur de risque de diabète de type 2 [10]. Ces résultats sont confirmés par les études de modèles animaux montrant sans ambiguïté que le déficit en adiponectine s'accompagne d'une insulinorésistance et d'une grande susceptibilité à l'athérosclérose. À l'inverse, l'administration d'adiponectine chez des souris lipodystrophiques ou obèses corrige leur insulinorésistance [11]. Les études familiales mesurant l'héritabilité de l'adiponectinémie ont conclu à une contribution génétique de $42 \%$ à $70 \%$ à la variance de ce trait, ce qui est considérable et comparable à l'héritabilité de la masse grasse. D'autres études familiales menées dans plusieurs populations ont identifié un locus en 3 q27 lié au risque de diabète de type 2 et de maladie coronarienne [12, 13]. Ce locus module des traits quantitatifs liés à I'IMC et au syndrome métabolique [14]. La localisation du gène de l'adiponectine APMI dans cette région en fait un candidat «positionnel» du syndrome d'insulinorésistance. Des polymorphismes génétiques (single nucleotide polymorphisms, SNP) ou variants du gène APMl inégalement prévalents dans l'espèce humaine pourraient en partie expliquer les différences individuelles dans la modulation de l'adiponectinémie, et donc contribuer au risque génétique de diabète de type 2 .

Un premier criblage partiel du gène à la recherche de SNP a été réalisé chez des sujets japonais [15]: une mutation silencieuse $\mathrm{Gl} 5 \mathrm{G}$ de l'exon 2 et une mutation faux-sens R112C de l'exon 3 ont été décrites sans qu'aucune corrélation ne soit établie avec l'adiponectinémie ou la pathologie. Ultérieurement, le criblage réalisé au sein de la population française d'une partie de 7,6 kb du gène incluant des séquences 5 ', les séquences codantes et en partie non codantes (introns et UTR), a mis en évidence 16 SNP également présents dans la population japonaise [16] (Figure 1). Le génotypage des SNP fréquents dans de grandes cohortes a ensuite révélé l'association entre certains variants du gène et des phénotypes associés au syndrome d'insulinorésistance (Tableau 1). Même si les résultats ne sont pas rigoureusement superposables d'une étude à l'autre, probablement en raison de différences ethniques, mais aussi dans les caractéristiques des populations étudiées (population générale, cohortes de sujets obèses...), ils ont en commun de mettre en évidence une relation étroite entre certains SNP du gène APMI et les taux d'adiponectine, l'insulinorésistance ou la présence d'un diabète de type 2. Comme ce risque n'est pas conféré par un SNP unique, mais par un haplotype formé par la combinaison délétère de plusieurs variants, il n'est pas aisé de déterminer quel est (ou quels sont) le (ou les) variant(s) fonctionnels(s). Ces SNP pourraient moduler le niveau d'expression du gène, ce qui est probable pour les SNP situés dans le promoteur du gène. Les SNP synonymes des régions codantes (mutations silencieuses) et les SNP introniques situés à proximité des exons pourraient quant à eux perturber l'épissage en modifiant les séquences correspondant à des enhancers ou à des silencers [20], ou encore moduler la stabilité de I'ARNm. Certains résultats préliminaires semblent conforter cette hypothèse, mais il est toujours possible que ces variants soient de simples marqueurs génétiques en déséquilibre de liaison avec des variants fonctionnels qu'il reste à mettre en évidence.

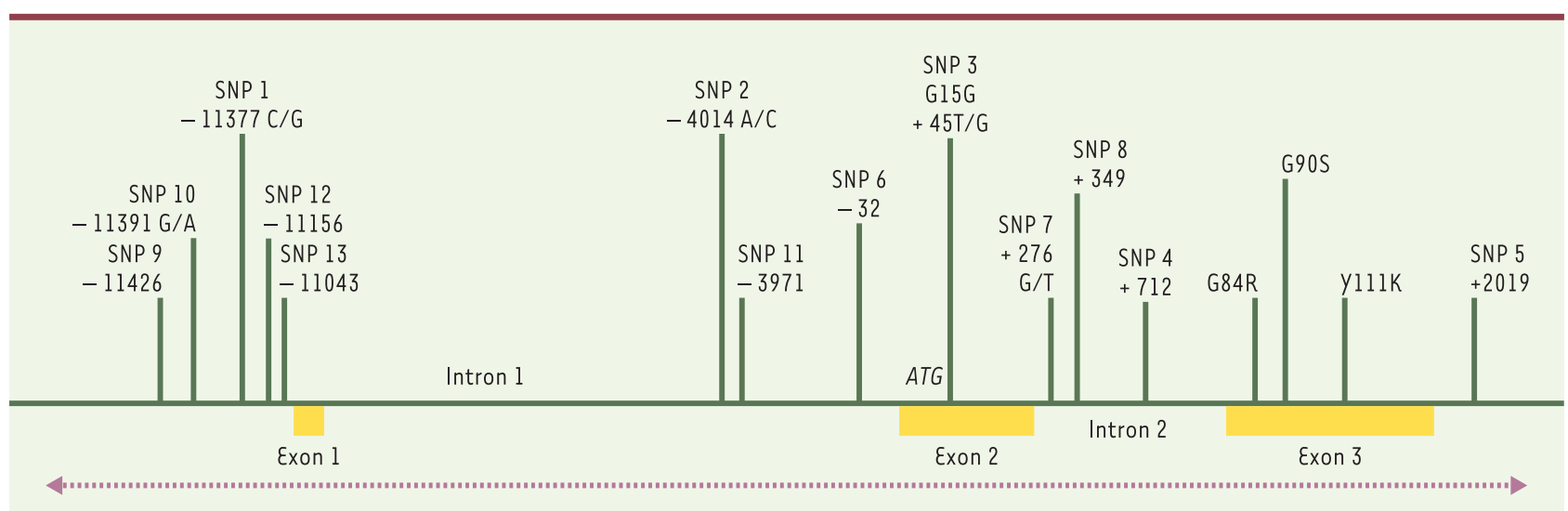

Figure 1. Organisation génomique et position des variants génétiques détectés dans le gène APMI. Les positions sont référencées par rapport au codon d'initiation ATG situé dans l'exon 2. SNP: single nucleotide polymorphisms. 
Parallèlement aux variants fréquents, neuf mutations situées dans la partie codante, toutes dans l'exon $3 \mathrm{du}$ gène (Figure 2), ont été étudiées dans les populations japonaise et française $[16,21]$. Les mutations non synonymes en position carboxyterminale (R221S, H24lP) n'ont montré d'incidence ni sur le taux d'adiponectine, ni sur les phénotypes associés au syndrome d'insulinorésistance. En revanche, les mutations G84R, G90S, Y111H, R112C et I164T sont fortement associées à des valeurs basses d'adiponectinémie et à un risque deux fois plus élevé de diabète de type 2. Ces mutations affectent le domaine collagène ou la jonction entre les domaines collagène et globulaire. Cette région très conservée entre les espèces inclut quatre résidus lysine $(68,71,80$ et 104$)$ et un résidu proline 94 , qui subissent les modifications post-traductionnelles (hydroxylation, glycosylation) requises pour l'oligomérisation indispensable à l'activité biologique de la protéine [22]. La protéine sauvage présente 22 répétitions Gly-X-Y ou Gly-X-Pro qui forment une triple hélice de collagène. Les mutations en positions 84 et 90 réduisent respectivement à 14 et 16 ce nombre de répétitions, altérant ainsi la triple hélice de collagène [16]. Les mutations jouxtant les domaines globulaire et collagène $(\mathrm{y} 111 \mathrm{H}, \mathrm{R} 112 \mathrm{C})$ pourraient altérer la configuration spatiale et donc l'oligomérisation. Ces mutations dans les parties codantes apparaissent comme des déterminants génétiques modulant l'adiponectinémie et le risque de diabète de type 2. Un effet synergique des variants dans le promoteur et des mutations dans les parties codantes a pu être démontré sur la variance du taux d'adiponectine [16].

L'effet de l'adiponectine dans la régulation de l'homéostasie énergétique a été le plus étudié : elle induirait un accroissement de l'oxydation des acides gras libres (AGL) [23] et de la dissipation d'énergie par le muscle, conduisant à une diminution du contenu musculaire, mais aussi hépatique, en triglycérides [11]. L'adiponectine permettrait également d'augmenter la capture musculaire du glucose et de diminuer sa production par le foie (principale anomalie responsable de l'hyperglycémie postprandiale des diabétiques), sans variation directe des taux plasmatiques d'insuline. Tous ces effets entraîneraient une amélioration des paramètres majeurs de l'homéostasie glucidique (capture de glucose, production hépatique de glucose) non pas à travers un effet sur la sécrétion d'insuline, mais par la potentialisation de ses effets tissulaires.

Il a été récemment montré que l'adiponectine, tout comme la leptine, stimule l'AMPK (AMP activated protein kinase) au niveau musculaire [24, 25]. L'isoforme $\beta$ de la coenzyme A carboxylase (ACC $\beta$ ), majoritaire dans le muscle, est connue pour être un régulateur de l'oxydation des acides gras. L'activation de l'AMPK par la

\begin{tabular}{|c|c|c|c|c|c|c|c|}
\hline & Associations & $\begin{array}{l}\text { Population } \\
\text { japonaise [17] }\end{array}$ & $\begin{array}{l}\text { Population } \\
\text { japonaise }\end{array}$ & $\begin{array}{l}\text { Population } \\
\text { française [16] }\end{array}$ & $\begin{array}{l}\text { Population } \\
\text { française }\end{array}$ & $\begin{array}{l}\text { Population } \\
\text { italienne [18] }\end{array}$ & $\begin{array}{l}\text { Population } \\
\text { allemande [19] }\end{array}$ \\
\hline \multirow{3}{*}{$\begin{array}{l}\text { - } 11391 \mathrm{G}>\mathrm{A} \\
\text { séquence } 5\end{array}$} & Avec le DT2 & Non & Non & Oui & NT & Non & NT \\
\hline & Avec l'adiponectinémie & Non & Non & Oui & Oui & Non & NT \\
\hline & Avec l'insulinorésistance & Non & Non & Non & Tendance & Non & NT \\
\hline \multirow{3}{*}{$\begin{array}{l}\text { - } 11377 \text { C>G } \\
\text { séquence 5' }\end{array}$} & Avec le DT2 & Tendance & Oui & Oui & NT & Non & NT \\
\hline & Avec l'adiponectinémie & NT & NT & Oui & Oui & Non & NT \\
\hline & Avec l'insulinorésistance & NT & NT & Non & Oui & Non & NT \\
\hline \multirow{3}{*}{$\begin{array}{l}+45 T>G \\
\text { exon } 2\end{array}$} & Avec le DT2 & Oui & Non & Non & NT & NT & NT \\
\hline & Avec l'adiponectinémie & Oui & NT & Non & Non & Non & NT \\
\hline & Avec l'insulinorésistance & Oui & NT & Non & Non & Oui & Oui \\
\hline \multirow{3}{*}{$\begin{array}{l}+276 \mathrm{G}>\mathrm{T} \\
\text { intron } 2\end{array}$} & Avec le DT2 & Oui & Non & Non & NT & NT & NT \\
\hline & Avec l'adiponectinémie & Oui & NT & Non & Non & Non & NT \\
\hline & Avec l'insulinorésistance & Oui & NT & Non & Non & Oui & NT \\
\hline
\end{tabular}

Tableau I. Associations détectées dans diverses populations entre les variants fréquents du gène APMI et les phénotypes du syndrome d'insulinorésistance. DT2: diabète de type 2 ; NT: non testél. Les résultats des secondes populations japonaise et française sont des communications personnelles. 
contraction musculaire inhibe l'ACC $\beta$ par phosphorylation, induisant ainsi la diminution du contenu en malonylCoA, et une dérépression de l'activité de la carnitine palmitoyl transférase 1 qui provoque l'augmentation de l'oxydation des AGL [26]. L'AMPK stimule la capture du glucose par la cellule musculaire [27] et diminue l'expression de facteurs impliqués dans la néoglucogenèse comme la phosphoénolpyruvate carboxykinase et la glucose 6-phosphatase hépatiques. Dans ce contexte, l'hypothèse de l'activation de l'AMPK par l'adiponectine a l'avantage de rendre compte, à elle seule, de la dualité des effets sur l'homéostasie glucidique : potentialisation des effets de l'insuline par augmentation de l'insulinosensibilité périphérique, et diminution de la production hépatique de glucose. Il est d'ailleurs remarquable que l'exercice physique, la metformine et les glitazones exercent également une partie de leurs effets antidiabétiques grâce à leur stimulation de l'oxydation des acides gras via la voie AMPK (Figure 3 ).

II reste beaucoup d'inconnues dans le mode d'action de l'adiponectine. Si cette hormone ne semble pas avoir d'effet direct sur la cellule $\beta$ (l'incubation d'îlots de Langerhans avec de l'adiponectine ne modifie en particulier pas la sécrétion d'insuline en réponse au glucose), d'autres effets sur la balance énergétique, en particulier centraux, ne sont pas à exclure. De plus, l'expression du gène $A P M I$ et la sécrétion d'adiponectine semblent hautement régulées par l'état métabo- lique : le TNF $\alpha$, en particulier, est un puissant inhibiteur de l'expression d'APM1, ce qui pourrait expliquer I’hypoadiponectinémie associée à l'obésité.

\section{Quels effets périphériques pour la leptine?}

La leptine est également une cytokine produite principalement par le tissu adipeux, dont le rôle majeur est d'assurer un contrôle à long terme de la masse graisseuse. Sa sécrétion est proportionnelle à la masse de tissu adipeux et ses effets sont principalement centraux : la liaison de la leptine sur son récepteur hypothalamique réduit la prise alimentaire et modifie le tonus du système nerveux autonome, agissant ainsi sur la sécrétion de l'insuline, la production hépatique de glucose et le métabolisme glucolipidique musculaire. Comme il existe des récepteurs de la leptine fonctionnels au niveau des tissus périphériques insulinosensibles, une action directe de cette hormone a été suggérée, mais les résultats des études sont contradictoires. II semble cependant que la leptine, tout comme l'adiponectine, soit capable de stimuler l'APMK et agir ainsi sur la concentration musculaire de malonyl-CoA et sur l'oxydation des acides gras [25]. Seules quelques rares mutations homozygotes de la leptine ou de son récepteur ont été décrites, qui résultent en une obésité massive liée à une hyperphagie majeure survenant dès les premières semaines de vie. Les patients concernés, en dépit d'une absence totale de signal leptine, ne présentent pas de syndrome métabolique exagéré (en comparaison de sujets obèses de même poids) et ne sont pas diabétiques. Aucune donnée convaincante ne suggère que des SNP fréquents de ces deux gènes (hormone et récepteur) contribuent au risque génétique de diabète de type 2 ou d'insulinorésistance.

TNF $\alpha$ et IL6: les duettistes de I'insulinorésistance

Le facteur de nécrose tumorale TNF $\alpha$ et l'interleukine 6 (IL6) sont des cytokines identifiées à l'origine comme des molécules pro-inflammatoires. Le TNF $\alpha$, produit
Figure 2. Mutations dans les parties codantes du gène APM1. Les mutations R112C, I164T, R221S et H241P (en rouge) ont été détectées dans la population japonaise. Les mutations G90S, R92X et Y111H (en bleu) ont été rapportées dans la population française. Seule la mutation G84R est commune aux deux populations. Ces résul-

tats soulignent les différences génétiques entre des populations d’origine différente.

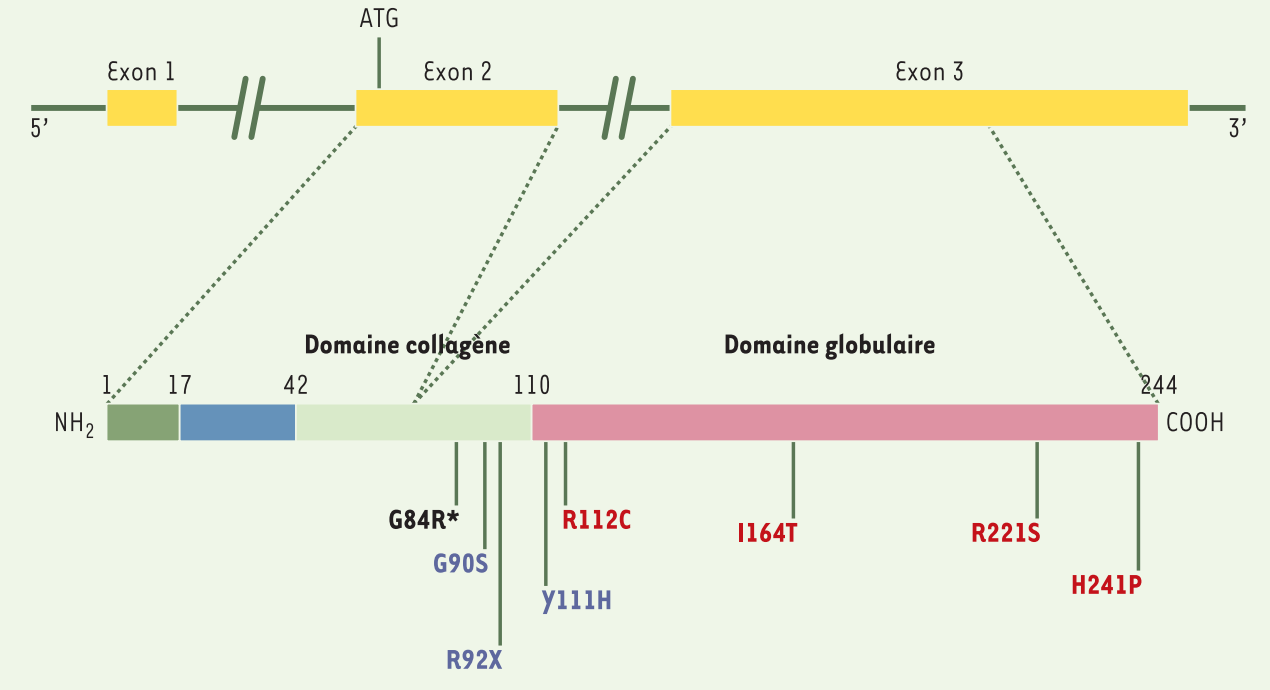


par les macrophages et les lymphocytes, a de nombreux effets : antitumoral, procoagulant, anorexigène et pyrogène. L'IL6, produite par les fibroblastes, les cellules endothéliales, les myocytes et les cellules endocrines, stimule la production des anticorps par les plasmocytes et la synthèse des protéines de phase aiguë par les hépatocytes. TNF $\alpha$ et IL6 sont aussi produits par les adipocytes [28]. Ces cytokines ont une action autocrine (dirigée vers les cellules sécrétrices), paracrine (vers les cellules voisines) et endocrine. Leur action autocrine et paracrine prédomine sur leur effet endocrine, ce dernier s'observant surtout en cas d'hypersécrétion (comme c'est le cas dans l'obésité).

Le TNF $\alpha$ est fortement surexprimé dans divers modèles animaux d'obésité et chez des patients obèses insulinorésistants [26]. Chez le rat obèse, la neutralisation du TNF $\alpha$ résulte en une amélioration de la sensibilité à l'insuline et une augmentation de la capture périphérique de glucose [29]. Cependant, cet effet n'a pas été mis en évidence chez l'homme. Chez les sujets obèses, les concentrations en TNF $\alpha$ sont élevées et associées à des marqueurs d'obésité et d'insulinorésistance.

Environ $30 \%$ de l'IL6 sécrétée est issue du tissu adipeux.

L'IL6 plasmatique croît proportionnellement avec le développement de l'obésité [30], et les études épidémiologiques en font un facteur de risque d'athérosclérose. Cela s'explique, d'une part, par des concentrations d'IL6 circulante étroitement corrélées à l'insulinorésistance chez l'homme sain ou obèse et, d'autre part, par le fait que I'IL6 contribue à l'hypercoagulabilité du syndrome métabolique par le biais de la stimulation dans le foie des protéines de phase aiguë (protéine C-réactive, fibrinogène et haptoglobine) et de la libération de molécules d'adhésion endothéliale.

Le TNF $\alpha$ et I'IL6 pourraient intervenir dans les mécanismes moléculaires de l'insulinorésistance et de l'athérosclérose qui lui est associée. L'insulinorésistance induite par le TNF $\alpha$ pourrait être indirecte, par augmentation des taux d'AGL, mais aussi directe, par blocage des voies de transmission du signal insuline en inhibant l'activité tyrosine kinase du récepteur de l'insuline [26]. Le TNF $\alpha$ bloque également la capture de glucose par régulation négative de l'expression du gène du transporteur de glucose GLUT4 [31]. Par ailleurs, le TNF $\alpha$ exercerait des effets délétères sur la fonction de la cellule $\beta$ pancréatique en augmentant sa sensibilité aux effets glucolipotoxiques à travers la production de radicaux libres [32]. L'implication du TNF $\alpha$ dans l'insulinorésistance a été confirmée chez la souris obèse $(o b / a b)$ invalidée pour le TNF $\alpha$ ou pour ses récepteurs, les résultats montrant des concentrations réduites d'AGL et une amélioration de la sensibilité à l'insuline.

Il est cependant difficile de déterminer si l'augmentation des niveaux de TNF $\alpha$ et d'IL6 sont la cause ou bien la conséquence de l'insulinorésistance induite par l'obésité. À la différence de la leptine, dont l'effet métabolique maximal se réalise dans l'organisme de poids normal, l'effet délétère du TNF $\alpha$ survient lorsque l'animal commence à devenir obèse. Cependant, l'hyperproduction de TNF $\alpha$ par l'adipocyte du sujet obèse inhibe la synthèse des triglycérides dans les adipocytes, limitant I'hypertrophie adipocytaire. Si la participation du TNF $\alpha$ et de I'IL6 au syndrome métabolique ne fait guère de doute, leur contribution au risque génétique de diabète de type 2 et d'insulinorésistance reste discutée. Le rôle de divers SNP situés dans le promoteur du gène du TNF $\alpha$ a été étudié dans plusieurs populations. Ainsi, le SNP -308 G>A semble associé à une augmentation des niveaux de TNF $\alpha$ circulant et du risque de diabète de type 2, mais pas de la mortalité cardiovasculaire $[33,34]$. Les porteurs de l'allèle -308 A sont par ailleurs plus fréquemment obèses que les sujets non porteurs. De plus, ce SNP a été associé aux paramètres d'insulinorésistance dans des populations européennes obèses, coronariennes ou souffrant de diabète de type 2. Quant au gène de l'IL6, aucune association entre le SNP $-174 \mathrm{G}>\mathrm{C}$ du promoteur et le développement d'un

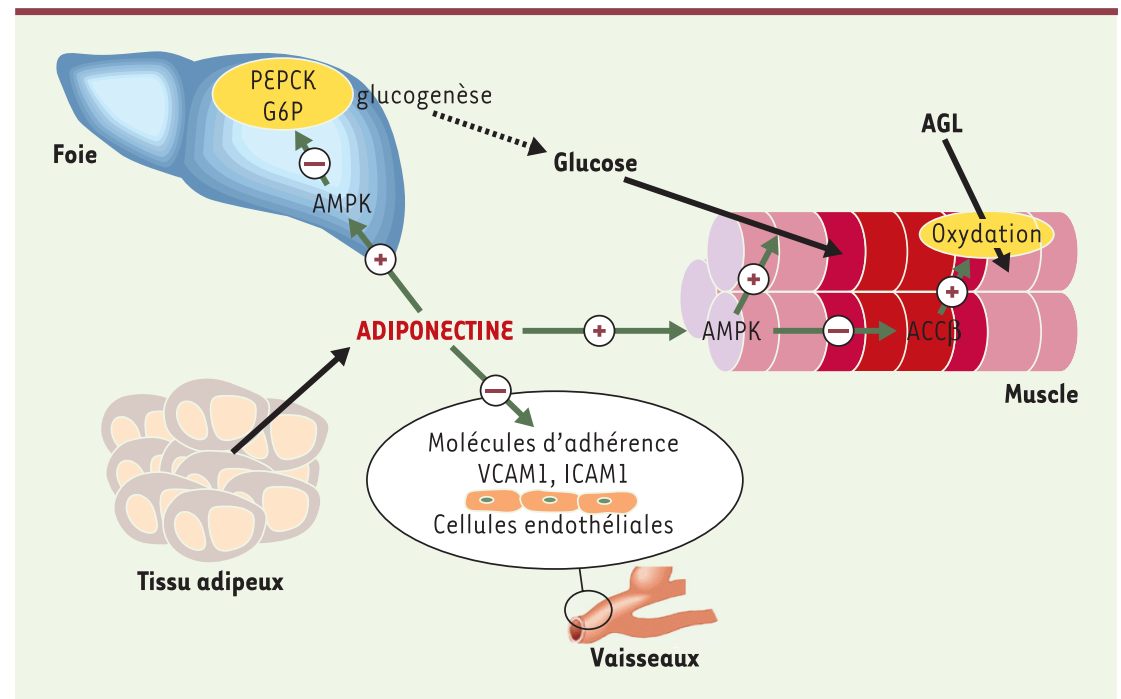

Figure 3. Rôle de l'adiponectine dans l'homéostasie glucidique, l'oxydation des acides gras libres (AGL) et les mécanismes anti-athérosclérotiques. PEPCK: phosphoénolpyruvate carboxykinase; G6P: glucose 6-phosphate; VCAM, ICAM: vascular et intercellular cell adhesion molecule; AMPK: AMP activated protein kinase; $A C C \beta$ : isoforme $\beta$ de la coenzyme A carboxylase . 
diabète de type 2 n'a été mise en évidence dans la population japonaise [35]. En revanche, dans la population espagnole, les porteurs de l'allèle -174 G en association avec une sécrétion élevée d'IL6 étaient plus souvent hyperlipémiques. Ce SNP semblait y moduler la sensibilité à l'insuline et la glycémie.

\section{Résistine : une hormone murine en quête de légitimité humaine}

La résistine est sécrétée par le tissu adipeux. Les concentrations sériques de résistine sont augmentées chez la souris obèse [36]. Chez ces animaux, l'administration d'un anticorps anti-résistine corrige les niveaux de glucose circulant et améliore la sensibilité à l'insuline. Mais l'administration de résistine recombinante n'entraîne qu'une intolérance au glucose. À l'inverse, la diminution de l'expression de la résistine est associée à l'augmentation de la sensibilité à l'insuline chez la souris. Chez l'homme sain, l'expression du gène de la résistine est très faible [37]. Ainsi, le concept de «résistine»: hormone principalement inductrice d'insulinorésistance, reste à confirmer. Les premiers résultats d'études génétiques réalisées au sein de plusieurs populations sont contradictoires et ne vont pas dans le sens d'une contribution forte du gène de la résistine au syndrome métabolique humain.

Le TNF $\alpha$, inhibiteur puissant de l'expression du gène de l'adiponectine, a la capacité de stimuler la production d'IL6 et de résistine, dont les effets s'opposent à ceux de l'adiponectine. Ainsi, pour mieux prendre en compte les susceptibilités génétiques individuelles, il serait nécessaire d'étudier l'interaction de ces quatre gènes.

\section{Les adipokines au cœur du syndrome métabolique}

L'ensemble des données disponibles suggère donc un rôle métabolique important de ces cytokines, qui non seulement interagissent directement avec les voies de transmission du signal insulinique, mais modulent également le métabolisme des acides gras. II faut cependant différencier l'état physiologique (organisme jeune, mince et en bonne santé) de la situation pathologique (obésité avec toutes ses conséquences). Dans le premier cas, il est probable que la faible sécrétion de TNF $\alpha$ et d'IL6 (voire d'autres cytokines de même type) limite leurs effets délétères à la sphère autocrine ou paracrine. Il se peut même que leur production soit alors bénéfique, du moins si l'on suppose que la survie prolongée et la résistance à l'obésité observées chez les souris dont le récepteur à l'insuline a été invalidé spéci- fiquement dans les adipocytes est généralisable à d'autres états d'insulinorésistance «adipocytaire». Parallèlement, la forte sécrétion d'adiponectine et l'efficience du signal leptine assurent l'oxydation des acides gras musculaires, évitant les dépôts graisseux ectopiques tout en contribuant à un blocage parfait de la production hépatique postprandiale de glucose.

Tout change en situation d'excès pondéral, surtout quand l'obésité est viscérale. L'hypersécrétion de TNF $\alpha$ et d'IL6 dans la circulation entraîne des effets endocrines indésirables qui aggravent l'insulinorésistance. La production d'adiponectine s'effondre et la résistance à la leptine s'installe, aggravant ainsi l'insulinorésistance. Un cercle vicieux d'aggravation du syndrome métabolique se met en place, qui touche désormais le système nerveux central (interruption des signaux produits par la leptine et l'insuline sur les centres de régulation énergétique et leurs projections du système nerveux autonome) et les cellules $\beta$ pancréatiques (perturbation de la sécrétion de l'insuline par glucolipotoxicité, mais aussi diminution progressive des capacités à former de nouveaux îlots de Langerhans). Quand la demande insulinique croissante dépasse les capacités de compensation pancréatique, la glycémie commence à s'élever, principalement après les repas, ce qui a des effets dévastateurs sur la fonction $\beta$ pancréatique et précipite l'apparition d'un diabète de type 2 qui devient rapidement irréversible.

La contribution des adipokines au diabète de type 2 est-elle primitive, ou simplement secondaire à l'excès de masse grasse? La génétique permet en partie de répondre à la question, du moins pour l'adiponectine. II semble bien que des polymorphismes génétiques du gène $A P M I$ non seulement diminuent l'adiponectinémie, mais sont associés au risque génétique de diabète de type 2. Comme la présence d'une adiponectinémie élevée semble protéger de la survenue de diabète (risque relatif 0,60 ) [10], l'hypoadiponectinémie induite à la fois par les facteurs génétiques, et environnementaux (dont l'hyperproduction de TNF $\alpha$ et de glucocorticoïdes) pourrait être un facteur important de la transition de l'obésité vers le diabète, et du syndrome métabolique vers la maladie coronarienne précoce. $\diamond$

\section{SUMMARY}

Adipocytokins, obesity and development

of type 2 diabetes

Normal metabolic balance is maintained by a complex homeostatic system involving multiple tissues and organs. Acquired or inherited defects associated to environmental factors in any part of this system can 
lead to metabolic disorders such as the syndrome $X$ which is presently a frequent syndrome in industrialized countries. It is characterized by a cluster of risk factors of atherosclerosis including insulin resistance, hyperinsulinemia, impaired glucose tolerance or type 2 diabetes, hypertension, dyslipidemia, and coagulation abnormalities. Its pathophysiology is likely to involve insulin resistance at the level of both skeletal muscle and visceral adipose tissue and altered fluxes of metabolic substrates between these tissues that in turn impair liver metabolism. Therapeutic intervention favours at present diet and exercise prescriptions. In addition, if necessary, specific treatment of the metabolic disorders is required. In the treatment of insulin resistance, new promising drugs are likely to be used in the next future. In this regard, adipose tissue, once thought to function primarily as a passive depot for the storage of excess lipid, is now understood to play a much more active role in metabolic regulation, secreting a variety of metabolic hormones and actively functioning to prevent deleterious lipid accumulation in other tissues and to modulate the insulin resistance. Here, we review new advances in our understanding of mechanisms leading to insulin resistance and type 2 diabetes from the perspective of the role and interactions of recently identified adipocyte-specific chemical messengers, the adipocytokines, such as adiponectin, tumor necrosis factor-alpha, interleukin 6 , and resistin. $\Delta$

\section{RÉFÉRENCES}

1. Scherer PE, Williams $S$, Fogliano M, Baldini G, Lodish HF. A novel serum protein similar to $\mathrm{Clq}$, produced exclusively in adipocytes. J Biol Chem $1995 ; 270$ : 26746-9.

2. Nakano $Y$, Tobe T, ChoiMiura NH, Mazda T, Tomita M. Isolation and characterization of GBP28, a novel gelatin-binding protein purified from human plasma. J Biochem 1996 ; $120: 803-12$.

3. Weyer C, Funahashi T, Tanaka S, et al. Hypoadiponectinemia in obesity and type 2 diabetes : close association with insulin resistance and hyperinsulinemia. J Clin Endocrinol Metab 2001 ; $86: 1930-5$.
4. Hotta K, Funahashi T, Arita $y$, et al. Plasma concentrations of a novel, adipose-specific protein, adiponectin, in type 2 diabetic patients. Arterioscler Thromb Vasc Biol 2000 ; 20 : 1595-9.

5. Yokota T, Oritani K, Takahashi l, et al. Adiponectin, a new member of the family of soluble defense collagens, negatively regulates the growth of myelomonocytic progenitors and the functions of macrophages. Blood 2000 ; 96 : 1723-32.

6. Ouchi N, Kihara S, Arita Y, et al. Adiponectin, an adipocyte-derived plasma protein, inhibits endothelial NF-kappaB signaling through a cAMPdependent pathway. Circulation 2000 ; 102 : 1296-301.

7. Ouchi N, Kihara S, Arita Y, et al. Adipocyte-derived plasma protein, adiponectin, suppresses lipid accumulation and class A scavenger receptor expression in human monocyte-derived macrophages. Circulation $2001 ; 103$ : 1057-63.

8. Kubota N, Terauchi $y$, Yamauchi T, et al. Disruption of adiponectin causes insulin resistance and neointimal formation. J Biol Chem 2002 ; 277 : 25863-6.

9. Stefan N, Vozarova B, Funahashi T, et al. Plasma adiponectin concentration is associated with skeletal muscle insulin receptor tyrosine phosphorylation, and low plasma concentration precedes a decrease in whole-body insulin sensitivity in humans. Diabetes 2002 ; $51: 1884-8$.

10. Lindsay RS, Funahashi T, Hanson RL, et al. Adiponectin and development of type 2 diabetes in the Pima Indian population. Lancet 2002 ; $360: 57-8$.
11. Yamauchi T, Kamon J, Waki $\mathrm{H}$, et al. The fat-derived hormone adiponectin reverses insulin resistance associated with both lipoatrophy and obesity. Nat Med 2001 ; 7 : 941-6.

12. Vionnet $\mathrm{N}$, Hani $\varepsilon l-H$, Dupont $S$, et al. Genomewide search for type 2 diabetessusceptibility genes in french whites : evidence for a novel susceptibility locus for early-onset diabetes on chromosome 3q27-qter and independent replication of a type 2-diabetes locus on chromosome lq21-q24. Am J Hum Genet 2000 ; $67: 1470-80$.

13. Francke S, Manraj M, Lacquemant C, et al. A genome-wide scan for coronary heart disease suggests in Indo-

Mauritians a susceptibility locus on chromosome $16 \mathrm{p} 13$ and replicates linkage with the metabolic syndrome on 3q27. Hum Mol Genet 2001 ; $10: 2751-65$.

14. Kissebah AH, Sonnenberg GE, Myklebust J, et al. Quantitative trait loci on chromosomes 3 and 17 influence phenotypes of the metabolic syndrome. Proc Natl Acad Sci USA $2000 ; 97: 14478-83$.

15. Takahashi M, Arita $Y$, Yamagata K, et al. Genomic structure and mutations in adipose-specific gene, adiponectin. Int J Obes Relat Metab Disord 2000 ; $24: 861-8$.

16. Vasseur F, Helbecque $N$, Dina C, et al. Singlenucleotide polymorphism haplotypes in the both proximal promoter and exon 3 of the APMl gene modulate adipocytesecreted adiponectin hormone levels and contribute to the genetic risk for type 2 diabetes in French Caucasians. Hum Mol Genet 2002 ; 11 : 2607-14. 
17. Hara K, Boutin P, Mori $Y$, et al. Genetic variation in the gene encoding adiponectin is associated with an increased risk of type 2 diabetes in the Japanese population. Diabetes 2002 ; 51 : 536-40.

18. Menzaghi C, Ercolino T, Di Paola R, et al. A haplotype at the adiponectin locus is associated with obesity and other features of the insulin resistance syndrome. Diabetes 2002 ; $51: 2306-12$.

19. Stumvoll M, Tschritter 0 , Fritsche A, et al. Association of the T-G polymorphism in adiponectin (exon 2) with obesity and insulin sensitivity : interaction with family history of type 2 diabetes. Diabetes 2002 ; 51 : 37-4l.

20. Cartegni L, Chew SL, Krainer AR. Listening to silence and understanding nonsense : exonic mutations that affect splicing. Nat Rev Genet 2002 ; 3 : 285-98.

21. Kondo H, Shimomura I, Matsukawa Y, et al. Association of adiponectin mutation with type 2 diabetes: a candidate gene for the insulin resistance syndrome. Diabetes 2002 ; $51: 2325-8$.

22. Wang $Y, X_{u} A$, Knight $C, X u$ LY, Cooper GJ. Hydroxylation and glycosylation of the four conserved lysine residues in the collagenous domain of adiponectin. Potential role in the modulation of its insulin-sensitizing activity. J Biol Chem 2002 ; 277 : 19521-9.
23. Fruebis J, Tsao TS, Javorschi $S$, et al. Proteolytic cleavage product of 30-kDa adipocyte complement-related protein increases fatty acid oxidation in muscle and causes weight loss in mice. Proc Natl Acad Sci USA 2001 ; 98 : 2005-10.

24. Yamauchi T, Kamon J, Minokoshi y, et al. Adiponectin stimulates glucose utilization and fatty-acid oxidation by activating AMP-activated protein kinase. Nat Med 2002 ; 8 : 1288-95.

25. Minokoshi Y, Kim YB, Peroni $0 D$, et al. Leptin stimulates fatty-acid oxidation by activating AMP-activated protein kinase. Nature 2002 ; 415 : 339-43.

26. Hotamisligil GS, Arner P, Caro JF, Atkinson RL, Spiegelman BM. Increased adipose tissue expression of tumor necrosis factoralpha in human obesity and insulin resistance. J Clin Invest 1995 ; 95 : 2409-15.

27. Mu J, Brozinick JT Jr, Valladares 0 , Bucan $M$, Birnbaum MJ. A role for AMP-activated protein kinase in contraction- and hypoxia-regulated glucose transport in skeletal muscle. Mol Cell 2001 ; 7 : 1085-94.

28. Fried SK, Bunkin DA, Greenberg AS. Omental and subcutaneous adipose tissues of obese subjects release interleukin-6: depot difference and regulation by glucocorticoid. J Clin Endocrinol Metab 1998 ; 83 : 847-50.
29. Hotamisligil GS, Shargill NS, Spiegelman BM. Adipose expression of tumor necrosis factoralpha : direct role in obesity-linked insulin resistance. Science 1993 ; 259: 87-91.

30. Mohamed-Ali V, Goodrick S, Bulmer K, Holly JM, Yudkin JS, Coppack SW. Production of soluble tumor necrosis factor receptors by human subcutaneous adipose tissue in vivo. Am J Physiol 1999 ; 277 : 971-5.

31. Hauner $H$, Petruschke T, Russ M, Rohrig K, Eckel J. Effects of tumour necrosis factor alpha (TNF alpha) on glucose transport and lipid metabolism of newlydifferentiated human fat cells in cell culture. Diabetologia 1995 ; 38 : 764-71.

32. Bolaffi JL, Rodd GG, Wang J, Grodsky GM.

Interrelationship of changes in islet nicotine adeninedinucleotide, insulin secretion, and cell viability induced by interleukin-1 beta. Endocrinology 1994 ; $134: 537-42$.

33. Mira JP, Cariou A, Grall F, et al. Association of TNF2, a TNF-alpha promoter polymorphism, with septic shock susceptibility and mortality : a multicenter study. JAMA 1999 ; $282: 561-8$.
34. Heijmans BT, Westendorp RG, Droog S, Kluft C, Knook $D L$, Slagboom PE.

Association of the tumour necrosis factor alpha 308G/A polymorphism with the risk of diabetes in an elderly population-based cohort. Genes Immun 2002 ; 3 : 225-8.

35. Hayakawa T, Takamura T, Hisada A, Abe T, Nomura G, Kobayashi K. IL-6 gene polymorphism - 174G/C does not contribute substantially to hyperlipidaemia and type II diabetes mellitus in Japanese men. Diabetologia 2002 ; $45:$ 453-4.

36. Way JM, Gorgun CZ, Tong $P$, et al. Adipose tissue resistin expression is severely suppressed in obesity and stimulated by peroxisome proliferatoractivated receptor gamma agonists. J Biol Chem 2001 ; $276: 25651-3$.

37. Haugen $F$, Jorgensen $A$, Drevon CA, Trayhurn P. Inhibition by insulin of resistin gene expression in 3T3-L1 adipocytes. FEBS Lett 2001 ; 507 : 105-8.

\section{TIRÉS À PART}

C. Lacquemant

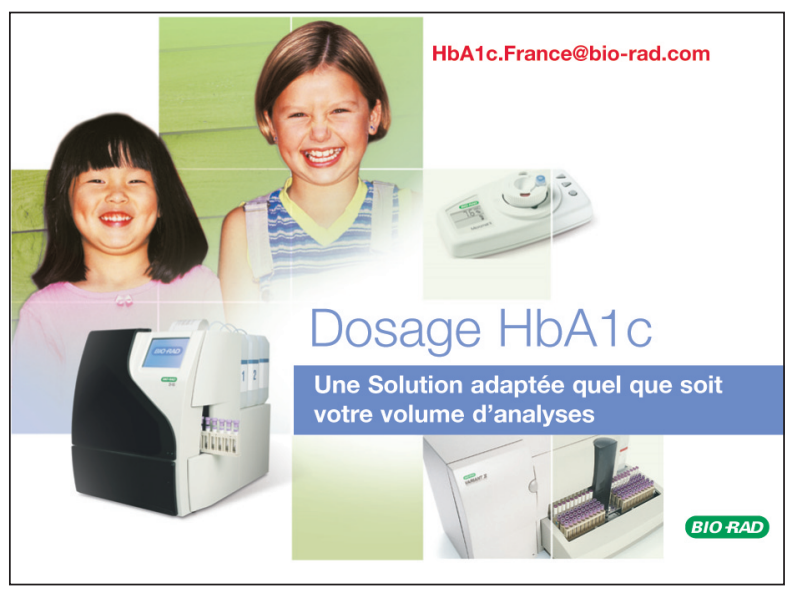

